\title{
EL TURISMO LITORAL EN EL MEDITERRÁNEO NOROCCIDENTAL: UNA APROXIMACIÓN CUALITATIVA A UN MODELO EN PROCESO DE TRANSFORMACIÓN ${ }^{1}$
}

\author{
LLUÍS CATALÀ OLTRA ${ }^{2}$ \\ Departamento de Sociología II \\ Universidad de Alicante
}

\begin{abstract}
Resumen
En este trabajo se caracteriza el momento actual y las perspectivas de futuro del turismo de costa en el Mediterráneo noroccidental (Islas Baleares, Cataluña y Comunidad Valenciana), una zona que ha alcanzado la madurez e incluso se ha enfrentado de manera exitosa a un hipotético declive. Para ello, se utilizan 33 entrevistas semiestructuradas a agentes clave del territorio (empresarios, responsables de la administración y académicos). A partir de su discurso se obtiene un diagnóstico de conjunto y se identifican las políticas (tanto empresariales como institucionales) a desarrollar para dar continuidad al buen momento del turismo del litoral mediterráneo español y, a más largo plazo, prevenir la decadencia del destino. Parece haber consenso en la necesidad de superar definitivamente el modelo anterior ligado al negocio inmobiliario, apostando por un turismo sostenible desde el punto de vista medioambiental, pero también sociocultural y económico; un tipo de turismo que, a través de la oferta complementaria y la singularización de la experiencia, agregue valor y contribuya al posicionamiento del producto en un contexto global altamente competitivo.
\end{abstract}

Palabras clave: Mediterráneo - turismo litoral - políticas - discurso - sostenibilidad

\begin{abstract}
In this work we characterise the Northwest Mediterranean (Balearic Islands, Catalonia and Valencian Community) coastal tourism's current moment and their future perspectives. It's a zone that has reached the maturity and even it has confronted with a hypothetical decline in a successful way. For this purpose, we use 33 semi-structured interviews to key agents of the territory (tourist company representatives, administration representatives and academicians). From their discourse we obtain a group diagnostic and we identify the developing politics (both company and institutional) to give continuity to the good moment of the Spanish Mediterranean littoral's tourism and, in a longer term, to prevent the destination's decadence. It seems to be a consensus in the need to surpass definitively the previous model tied to the real-estate business, and to bet on a sustainable tourism from the environmental point of view, but also from the sociocultural and economic. Through the complementary offer and the experience's singularization, it's a sort of tourism that adds value and contribute to the product's positioning in a highly competitive global context.
\end{abstract}

Key words: Mediterranean - coastal tourism - politics - discourse - sustainability

${ }^{1}$ Este escrito surge de una investigación para el Institut Ignasi Villalonga d'Economia i Empresa, con la financiación del Ayuntamiento de Barcelona y de las diputaciones de Barcelona y Girona. Asimismo, agradezco la lectura y comentarios de este texto a Josep A. Ivars (Instit. Univ. de Investigaciones Turísticas, Universidad de Alicante).

2 luis.catala@ua.es 


\section{INTRODUCCIÓN: LA TRANSFORMACIÓN DEL TURISMO DE COSTA}

En esta investigación, nos centramos en el tipo de turismo que tradicional y popularmente ${ }^{3}$ se ha denominado "sol y playa", aunque nosotros utilizaremos preferentemente el término "turismo de costa" o "turismo litoral", entendiendo que el concepto de "sol y playa" es insuficiente, porque en estas zonas ya se hacen otras muchas actividades que se suman a la tradicional de tomar el sol en la playa. Será precisamente la mutación de un modelo de paquetitzación limitado al disfrute de las playas hasta la actual diversificación de la oferta el que centre la atención de este trabajo.

El objetivo central de esta propuesta es caracterizar el momento actual y las perspectivas de futuro del turismo de costa en un área del Mediterráneo que ha alcanzado la madurez e incluso se ha enfrentado de manera exitosa al hipotético declive anunciado desde determinados posicionamientos ya clásicos (ver Butler, 1980). A partir del análisis de discurso de agentes claves del territorio, pretendemos determinar los problemas; a partir de ellos, identificar los retos de futuro; y, finalmente, desarrollar las actuaciones que permitan mantener el sector turístico en el mediterráneo noroccidental en niveles de excelencia. No se trata, como suele ser habitual, de desarrollar un aspecto o conjunto de aspectos concretos, sino de reflejar la globalidad de asuntos que afectan a la configuración de una oferta turística.

La desaceleración, y en algunos momentos estancamiento, desde final de los setenta hasta principios de los noventa, dio pie después al boom inmobiliario propiciado por la Ley del Suelo de 1998, que daría paso definitivamente al sobredimensionamiento de la planificación urbanística residencial (Burriel, 2009 y 2014), que a su vez se combinó con un fácil acceso a los recursos financieros (Demajorovic et al., 2011: 789). El territorio demostró que todavía tenía capacidad para exprimirse y la degradación del paisaje litoral todavía fue más notable, salvo excepciones donde se apostó por una relativa protección (el Alt Empordà en el norte de Cataluña y Menorca, por ejemplo). En este periodo y en general, se renuncia a "un modelo profesionalizado de producción turística atento a las cualidades del territorio" y, en cambio, hay una desviación progresiva, y en cierto sentido, crónica, hacia "las actividades relacionadas con la construcción inmobiliaria y los componentes especulativos asociados a la compraventa de suelo y viviendas" (Obiol y Pitarch, 2011: 178). Se pone de manifiesto la contradicción entre los intereses de un empresario turístico ofertante de servicios, que tiene que "permanecer en el territorio e implicarse a medio y largo plazo en su conservación y calidad" y el empresario promotor del turismo residencial, que en definitiva es un empresario del sector inmobiliario, y "que obtiene toda su rentabilidad en el momento de vender el bien", la vivienda (Fernández y Barrado, 2011: 377; ver también Aledo, 2008). ${ }^{4}$ En el caso del promotor inmobiliario, esto implica un comportamiento que algunos han calificado de "nómada" (Demajorovic et al., 2011: 790), en cuanto que su negocio se basa en el consumo de nuevos territorios.

\footnotetext{
${ }^{3}$ Nos referimos a una tradición popular (pero también académica) contemporánea, porque es un concepto que sólo se puede referir al que surge a partir de los años sesenta. Antes, habría que hablar más bien de "sol y mar" (Pérez y Navas, 2014: 260).

${ }^{4}$ De todos modos, eso no es incompatible con el hecho de que muchos empresarios turísticos se apuntaron también en aquellos momentos a la rentabilidad a corto plazo de la inversión inmobiliaria.
} 
Evidentemente, esto tiene como consecuencia un bajo compromiso con cada uno de los territorios por los cuales transita.

Esta fase ha condicionado enormemente la presente y es responsable de muchos de los retos a los que se enfrenta el sector y que trataremos de una manera u otra en este trabajo, como por ejemplo la sostenibilidad (Torres et al., 2013), la densificación del litoral (Horrach, 2014; Roca y Villares, 2014; Ramos, 2013), la amplia oferta ilegal de apartamentos (Ernst \& Young, 2015), la transformación de los destinos turísticos en ciudades (Rovira y Anton, 2014), etc. Éstas y otras cuestiones pendientes del sector se tienen que afrontar precisamente en uno de los mejores momentos para el turismo de territorios mediterráneos como las Islas Baleares, Cataluña y la Comunidad Valenciana, porque 2014, 2015, 2016 y 2017 han sido años excepcionales y las previsiones a corto plazo indican que el buen momento se mantendrá (OMT, 2016), salvo que persista la inestabilidad política en Cataluña o los atentados terroristas en este mismo territorio tengan un impacto notable.

El momento actual también es el del estadio máximo de la internacionalización de las economías y de las sociedades, y esto tiene desafíos evidentes en cuanto a la oferta turística, porque esta zona compite con una cantidad mayor de destinos, pero también en cuanto a la demanda: países que hasta hace poco apenas viajaban, ahora lo están haciendo masivamente y todos los destinos pugnan por atraerlos. Asimismo, la globalización significa acortamiento de las distancias vía tecnología (transportes, comunicaciones), y esto tiene implicaciones claras en el desarrollo del turismo, porque son claramente elementos facilitadores. Por eso, el turismo es una de las manifestaciones más evidentes de la globalización, con consecuencias sobre los espacios y las identidades, en la medida que, a pesar de su contribución a la diversidad, genera a la vez paisajes uniformizados y erosiona las particularidades de los territorios (Creus y Celada, 2011: 8; Muñoz, 2010; Anton, 2010: 161; Carvalho y Moquete, 2012: 447). Pero "el valor simbólico de los lugares -de los destinos- es el factor que genera expectativas a los turistas y los convierte en territorios con capacidad de atracción y de uso turístico", a pesar de que "se tiene que plantear, (...) a modo de compromiso ético, que la identidad no puede ser (...) el elemento fundamental de una atracción ni convertirse sólo en un atractivo". Hay que respetar el paisaje y los elementos identitarios que genera "para que puedan ser leídos por los turistas, pero también para que sean útiles a sus propios habitantes, preservando valores ecológicos, productivos y sociales" (Anton, 2010: 158-159).

Conectando esto último con lo que comentábamos sobre la nueva demanda turística, los grandes volúmenes de emisión de turistas de las dos próximas décadas vendrán desde China y el resto de Asia (OMT, 2015; Córdoba, 2014: 10-11), precisamente lugares donde el sol y playa no es un producto apreciado. Si estamos con Salvador Anton (2010: 157) cuando apunta que "hay una premisa fundamental en el funcionamiento de cualquier sistema turístico que dice que son sus contenidos simbólicos los que lo dotan de identidad, lo diferencian y lo hacen visible" y considerando, como nos comenta en una entrevista Bea Ponce (Nihao \& You, empresa de adaptación al turismo chino), que uno de los elementos que más busca el turista asiático es la autenticidad, será vital la apuesta por toda una serie de actividades complementarias (gastronomía, patrimonio cultural, enoturismo, representaciones y 
recursos etnográficos, turismo activo, etc.), ${ }^{5}$ que hacen de la estancia en las costas una experiencia más enriquecedora y capaz de responder a una mayor variedad de perfiles.

Y si la mejora del transporte (y en los últimos años el abaratamiento de los vuelos) ha tenido un impacto visible en el turismo, también las tecnologías de la información y la comunicación han implicado cambios destacables en un lapso de tiempo muy breve (Córdoba, 2014; Ruiz y Tronch, 2013; Riera y Aguiló, 2009). Internet ya es un medio de primera magnitud para la búsqueda y contratación de viajes y experiencias turísticas (redes sociales, Tripadvisor, Trivago, Booking, Vueling, etc.), pero también para la comunicación y evaluación posterior de estas experiencias (nuevamente redes sociales, Tripadvisor, etc.). Asimismo, diferentes aplicaciones para smart phones (museos, transporte público, evaluadores de vinos y restaurantes, trazadores de rutas) facilitan la visita a cada vez más turistas. Y portales como Airbnb o Home Away han revolucionado la oferta de alojamientos, no sin polémica por la gran cantidad de viviendas no regladas que se ofrecen. Todo ello, ha obligado a las instituciones promotoras y a las empresas a adaptarse y destinar recursos al posicionamiento en Internet o en las redes sociales (Iglesias y Fernández, 2012), así como a buscar la presencia a través de APP.

Respecto a los portales de oferta de apartamentos, hay que puntualizar que en algunos casos los no reglados ya eran amplia mayoría antes de la llegada de estas páginas web (Cambrils y Nàcher, 2005: 121), pero es el canal el que amplifica el impacto y pone en peligro el negocio reglado. En la distribución todavía siguen dominando los turoperadores y los intermediarios clásicos, pero Internet lo está condicionando todo (Flores et al., 2011: 346-347), de forma que aquel "paquete estandarizado fordista" va perdiendo fuerza y los intermediarios se tienen que adaptar a la demanda de singularización. En cierto modo, se está produciendo un "proceso progresivo de desintermediación", que ya era visible a comienzos de la crisis (Riera y Aguiló, 2009: 127).

Por otro lado, según Santos et al. (2014: 74-76; ver también Ivars et al., 2013), son cuatro los factores que determinan la competitividad de los destinos turísticos maduros: infraestructuras, impacto medioambiental, vitalidad económica y gestión del destino $^{6}$. En el primer caso, evidentemente, hace falta que estén disponibles las infraestructuras adecuadas, incluyendo su modernización; por otro lado, la asunción de la madurez normalmente viene acompañada de la masificación, y esto puede operar en contra de la sostenibilidad del territorio y contribuir a la degradación del entorno; el destino también puede perder vitalidad económica por el descenso en el número de visitantes o la reducción de precios para captar más; y finalmente, se necesita una mejor coordinación de las instituciones gestoras del destino para hacer frente a un incremento de la diversificación y la complejidad de estos destinos. Los entrevistados para esta investigación han apuntado que las infraestructuras, en general, son adecuadas, pero hay que solucionar algunas carencias específicas, como por ejemplo la provisión y gestión de agua en las Islas Baleares, cuestión que tiene que ver también con la sostenibilidad (aunque es una carencia compatible con los esfuerzos paralelos de conservación del entorno); además, está claro que una buena parte de la franja litoral está densificada y el

\footnotetext{
${ }^{5}$ Por ejemplo, en un estudio con turistas internacionales en el destino costero del Algarve (Portugal), se ha constatado que junto con el clima y los atractivos naturales (playa y mar, básicamente), los visitantes aprecian los atractivos culturales y sociales (Santos et al., 2014: 77). Y son turistas que van, sobre todo, a disfrutar de las playas.

${ }^{6}$ Hay autores que prestan atención a otros factores, como por ejemplo las condiciones de la demanda, el estado de los sectores auxiliares o la dinámica empresarial (Marco et al., 2011: 71).
} 
paisaje ha perdido parte de su atractivo; se mantiene la vitalidad económica a pesar de la crisis, por el buen momento del turismo en el Mediterráneo español; y en el caso de la gobernanza, se están haciendo esfuerzos para adaptarse a lo que han implicado diez años de expansión inmobiliaria, pero, por otro lado, Salvador Anton (Universitat Rovira i Virgili) lamentaba en las entrevistas que los organismos responsables del turismo se dediquen en exceso a la promoción y poco a la definición de políticas, a la articulación de un modelo, una hoja de ruta para los destinos. Son retos que hay que afrontar y en este trabajo se esbozan algunas líneas de actuación para superar esta fase de madurez, y quizá pasar a una de rejuvenecimiento. ${ }^{7}$

Centrados en el factor medioambiental, está claro que hoy en día, y más después del periodo 1998-2008, ha pasado definitivamente a ser un elemento de primer orden en las políticas públicas, a pesar de que las tensiones con los agentes favorables a la liberalización del suelo puedan continuar y todavía algunos actores de la administración acaben cediendo a presiones y/o actuando como facilitadores. También los empresarios hoteleros tienen claro que la preservación del entorno discurre a favor del destino. El buen estado de los recursos naturales favorece el turismo activo y las actividades complementarias que pide un turista con patrones de consumo más complejos:

Los nuevos turistas ya no buscan tanto servicios como experiencias que satisfarán su sistema emocional -un hecho que nos tiene que guiar hacia la incorporación de activos físicos $e$ intangibles para el sistema de producción de estas experiencias, intentando preparar nuevos espacios para el descubrimiento, las relaciones y las sensaciones (Anton, 2009). El nuevo contexto impone un cambio de modelo hacia la diversificación de la oferta turística que va más allá del producto tradicional de sol y playa-los turismos cultural y activo están siendo redescubiertos y el patrimonio natural está siendo reevaluado como un objeto de consumo para vacaciones y ocio. (Roca, 2014: 345-346).

Por tanto, cobra mucha importancia "el concepto de resiliencia, definido desde la ecología como la habilidad para adaptarse o recuperarse de las presiones sociales, ambientales o económicas ejercidas sobre un sistema" (Roca y Villares, 2014: 494). Se trata de recuperar ecológica y socialmente el territorio para que pueda servir a este nuevo modelo de turismo que se apoya en el patrimonio natural y cultural, y así posibilitar un marco experiencial adecuado. ${ }^{8}$ En este sentido, la Costa Brava es ejemplo

\footnotetext{
${ }^{7}$ Se tienen documentadas algunas experiencias de adaptación todavía en la fase de bonanza para los casos de Benidorm y Gandía. Por ejemplo, en Gandía, en pleno auge inmobiliario, se buscó la mejora de la habitabilidad urbana, atenuar la presión constructora y apostar por la sostenibilidad, entre otras iniciativas (Cambrils y Nàcher, 2005: 117-120). En el caso de Benidorm, 2002-2007 fue un periodo en el cual se buscó una mayor diversificación del destino (Ivars et al., 2013: 188). El Plan de Marketing Turístico de Benidorm 2011-2015 apunta que "existe una clara necesidad de reconducir las percepciones negativas hacia Benidorm (sobre todo masificación e inexistencia de personalidad propia y claramente definida) y diferenciarse a partir de una mayor diversidad de ofertas de ocio y actividades que enriquecen el producto actual". El lema tiene que pasar por "constituirse en el principal referente de un destino de ocio de calidad con un buen ambiente y un clima privilegiado durante todo el año, en un entorno mediterráneo. (...) Se tiene que transmitir que siempre hay algo para ver o para hacer en un ambiente mediterráneo" (Innova Management, 2014: 114-115). Asimismo, y refiriéndose a la Playa de Palma, Biel Horrach afirma que "en su diversidad hay uno de los potenciales para la regeneración de los espacios turísticos maduros, a través de la recuperación y puesta en valor de los productos turísticos presentes en su propia forma urbana, dotando al destino de la suficiente diversidad de productos, principal motivación del turismo actual" (2014: 368).

${ }^{8}$ En definitiva, se trataría de conseguir un turismo sostenible en los términos que propone el Programa Medioambiental de Naciones Unidas: en primer lugar, "hacer un uso óptimo de los recursos
} 
de buenas y malas prácticas, porque, si bien globalmente no es una de las marcas turísticas más sostenibles de Cataluña (Torres et al., 2013: 63-64), especialmente por el desarrollo en el sur, la bahía de Roses y, en general, el Alt Empordà es uno de los entornos más protegidos y más atractivos para este nuevo tipo de visitante (Roca y Villares, 2014: 498-500 y 518-519). En cualquier caso, también en la comarca de la Selva, al sur de Girona, están trabajando en esta dirección, digamos, resiliente.

Será, pues, el turismo sostenible el más apto para ayudar al visitante a tener las experiencias que busca, aquellas que le impacten de tal manera que se plantee repetir otro año y, si acaso, llegar a ser fidelizado. Es un turista que está cambiando de hábitos (Riera y Aguiló, 2009: 127), que está ganando complejidad, que está afinando su capacidad de exigencia. Para darle respuesta, hace falta, pues, valorar el territorio "a través de la creación de argumentos recreativos -rutas interpretadas, ecomuseos, equipamientos de ciencia activa, parques de actividades o tematización del patrimonioque actúen como catalizadores de la actividad" (Anton, 2009: 98); hay que aprovechar la diversidad de la mayor parte de los destinos del litoral este español para generar estos productos y ofrecer una experiencia singular, que tiene que ser transmitida también de manera diferencial, buscando imágenes que sean la síntesis de este (nuevo) ${ }^{\mathbf{9}}$ concepto que transmiten (Novo et al., 2013: 44) y sirviéndose adecuadamente de la tecnología (redes sociales, aplicaciones para móviles, etc.). La excelencia reconocida de grandes cocineros como por ejemplo los hermanos Roca, Fernando P. Arellano o Quique Dacosta; la experiencia musical única en el mundo de Ibiza; el conjunto natural de Menorca, reserva de la biosfera; el parque Port Aventura, uno de los mejores de Europa; las doce fiestas valencianas de interés turístico internacional; o los quince patrimonios de la humanidad de esas tres comunidades autónomas, son sólo algunas de las puntas de lanza de un extenso producto complementario para dar respuesta a la demanda de singularidad.

\section{2. ÁMBITO GEOGRÁFICO}

El territorio sobre el cual centramos la atención son las comunidades autónomas de las Islas Baleares, Cataluña y la Comunidad Valenciana, que, en conjunto, es un lugar donde se ha producido un fuerte crecimiento poblacional en el período 1998-2008, especialmente en las Islas Baleares, núcleos como Lloret de Mar o Salou en Cataluña, o comarcas como las que lideran Benidorm o Torrevieja en la provincia de Alicante, por mencionar algunos casos significativos. Esto ha contribuido a la densificación urbana de la fachada litoral, no al nivel de las dos grandes áreas metropolitanas de esta zona (Barcelona y Valencia), pero sí de una manera que ha alterado decisivamente el paisaje. $\mathrm{Y}$ en estos crecimientos ha tenido mucho que ver el asentamiento de residentes

medioambientales que constituyen un elemento clave en el desarrollo turístico, manteniendo procesos ecológicos esenciales y ayudando a conservar el patrimonio natural y la biodiversidad", pero también "respetar la autenticidad sociocultural de las comunidades" y desarrollar acciones económicas que beneficien a todos los agentes del territorio y que generen empleo de manera sostenida (UNEP, 2013: 266). Las dos últimas cuestiones tienen una importancia que no se debe despreciar, pues la actitud de los residentes hacia el turismo es uno de los determinantes del desarrollo de los destinos (Gutiérrez, 2010).

${ }^{9}$ Ya en 2004, profesionales, empresarios y políticos se referían a la necesidad de añadir valor a la oferta de sol y playa, complementando con otras actividades y productos, como por ejemplo turismo activo, cultura, gastronomía, potenciar los recursos naturales, etc. (Hosteltur, 2004: 7 y 13). Por lo tanto, el momento actual lo único que hace es reafirmar esta apuesta, porque se ha detenido en parte el desarrollo urbanístico y estos valores ya no son una posible tendencia de futuro, son una necesidad presente para el sector. 
extranjeros de la Unión Europea, pero también la inmigración laboral atraída por el ritmo de crecimiento en el periodo de bonanza. Por ejemplo, en los cuatro grandes centros de atracción turística (Calvià, Salou, Lloret de Mar y Benidorm) se ha pasado de porcentajes alrededor del 10\% de extranjeros en 1998 a más del $30 \%$ en 2014. Evidentemente, esto impulsó el sector de la construcción hasta cifras inéditas en el volumen de viviendas y en la valorización de las propiedades (ver Català, 2016).

Se ha acotado una zona ciertamente con características comunes, pero entendemos que también se podría haber ampliado al resto del Mediterráneo español. De hecho, se evaluó la posibilidad de incluir la Región de Murcia, pero se renunció finalmente a su incorporación al estudio porque su oferta y demanda turísticas tienen características que le alejan de las otras tres comunidades autónomas. Por ejemplo, un nivel de pernoctaciones de extranjeros muy inferior al resto $(26 \%$ en hoteles, cuando el resto de comunidades supera el 45\%; en campings o apartamentos las diferencias son similares), una oferta hotelera también de menor entidad (apenas 6 habitaciones por 1.000 habitantes, cuando los otros territorios tienen más del doble), una inferior ocupación hotelera (por debajo del $50 \%$ en el conjunto de 2016, y menos del $70 \%$ en agosto de ese año; el resto de comunidades superan el $60 \%$ anual y el $80 \%$ en agosto; las distancias son aún más acusadas en campings), una estancia media de poco más de 2 días (en los otros territorios es al menos de 3 días) ${ }^{10}$ e indicadores de rentabilidad del sector hotelero también claramente por debajo (ver INE, 2016). Por tanto, con Murcia no podemos hablar de un nivel de madurez equiparable al de las Islas Baleares, Cataluña y la Comunidad Valenciana. De todos modos, entendemos que buena parte del diagnóstico y las propuestas que de este estudio se derivan son aplicables al turismo litoral murciano o andaluz.

Respecto a la oferta de alojamiento, en el Estado español, sólo las Islas Canarias y algún punto de la Costa del Sol llegan a la altura de los principales núcleos de esta terna de comunidades. A pesar de que la distribución está algo más equilibrada en apartamentos, en hoteles podemos hablar de una fuerte concentración en las Islas Baleares, y los entornos de estos grandes núcleos (Benidorm, Salou, Lloret de Mar). En cambio, en el caso de los campings, las Islas Baleares tienen una representación testimonial, y se destacan otras zonas, como por ejemplo el norte de la provincia de Girona o el sur de la de Valencia, junto a las infraestructuras situadas alrededor de los núcleos de gran atracción. Son lugares, todos ellos, donde la proporción de pernoctaciones de extranjeros es elevadísima -normalmente entre el 80 y el $90 \%$ (Català, 2016). Este hecho ha contribuido a su liderazgo en el conjunto mediterráneo:

\footnotetext{
${ }^{10}$ Todos los datos son de la Encuesta de Ocupación Hotelera, ocupación en campings, (INE, 2016).
} 


\section{Datos de pernoctaciones y camas en establecimientos alojativos en grandes regiones litorales de la UE mediterránea}

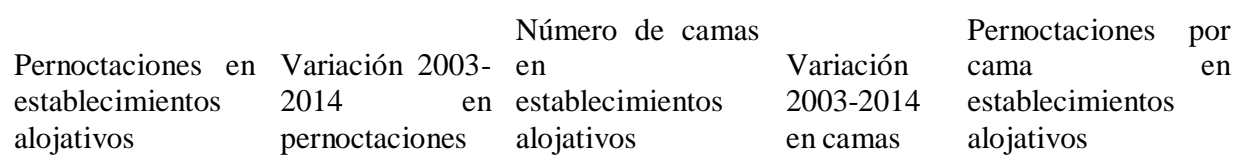

Litoral sur español ${ }^{(1)}$

Litoral este $^{\text {español }}{ }^{(2)}$

Litoral mediterráneo francés (3)

Litoral tirreno italiano ${ }^{(4)}$

Litoral adriático italiano $^{(5)}$

Balcanes adriáticos ${ }^{(6)}$

Grecia ${ }^{(7)}$

Islas-Estado mediterráneas ${ }^{(8)}$
62.012 .743

176.037.892

96.784 .961

141.826 .316

136.350.479

67.300 .131

91.534 .772

22.496 .290

20,1

15,5

552.969

34,7

1.580.868

12,4

111,4

50,7

1.318 .775

$-5,9$

1.836 .480

17,4

1.945 .478

12,8

70,1

Fuente: EUROSTAT y elaboración propia.

Notas: excepto para el Litoral este español, el Litoral sur español y Chipre, el resto de grandes regiones costeras mediterráneas tienen como último año con datos el 2013. Las grandes regiones que involucran a NUTS2 italianas tienen datos a partir de 2004, no de 2003.

(1) Litoral sur español incluye Andalucía y Murcia.

(2) La EURAM en este caso está representada por las Islas Baleares, Cataluña y el País Valenciano; Cataluña Norte (departamento de los Pirineos Orientales), con poco peso en las pernoctaciones, forma parte del Litoral continental mediterráneo del estado francés, porque los datos no se pueden fragmentar por NUTS3, que habrían permitido aislar Cataluña Norte de la región administrativa del Languedoc-Rossellón.

(3) El Litoral mediterráneo francés incluye las regiones del Languedoc-Rossellón, Provenza-Alpes-Costa Azul y la isla de Córcega.

(4) El Litoral tirreno italiano incluye las regiones de Liguria, Toscana, Lacio, Campania, Basilicata, Calabria, y las islas de Sicilia y Cerdeña.

(5) El Litoral adriático italiano incluye las regiones del Friuli-Venecia Julia, el Véneto, Emilia-Romaña, Las Marcas, Los Abruzos y Apulia.

(6) Los Balcanes adriáticos incluyen las regiones de Croacia Adriática y Eslovenia Occidental.

(7) Grecia incluye todas las regiones litorales continentales (Macedonia, Tesalia, Tracia, el Peloponeso, Epiro, Ática, etc.) y las islas (Jónicas, Cícladas, Dodecaneso, Creta, etc.).

(8) Las Islas-Estado mediterráneas incluyen Chipre y Malta.

La comparativa de estas ocho regiones euromediterráneas sitúa la zona de estudio en una posición claramente destacada en pernoctaciones, porque sus más de 176 millones superan aproximadamente en 35 millones la que le sigue en volumen, que es el litoral tirreno italiano. A partir de los litorales italianos, el resto, encabezado por el litoral mediterráneo del Estado francés o Grecia, no llegan siquiera a 100. Por otro lado, la madurez de los destinos turísticos del área de estudio hace que su demanda haya 
registrado uno de los crecimientos más bajos, y más en contraste con el este (los Balcanes adriáticos y Grecia).

Respecto a la oferta, el liderazgo del litoral este español está contestado por los litorales italianos y el del Estado francés. Y en la variación de la oferta 2003-2014 pasa algo similar a lo que hemos detectado en la demanda, con crecimientos exiguos en la parte occidental y más notables en el Mediterráneo oriental. Finalmente, la relación oferta-demanda indica que sólo las islas-Estado, Chipre y Malta, se encuentran claramente por encima de los territorios españoles en la ratio de pernoctaciones por cama; Grecia, Francia, Italia o Croacia están lejos de obtener el rendimiento que recogen los destinos del Estado español. En definitiva, se trata de una zona líder, que mantiene su vigor a pesar de haber alcanzado la madurez y más aún los últimos cuatro años.

\section{APUNTES METODOLÓGICOS}

En este trabajo se plantea la aproximación a las opiniones de algunos de los que hemos considerado agentes clave desde un punto de vista académico, empresarial e institucional. Evidentemente, el discurso gira en torno al turismo litoral y sus retos; en este sentido, las conversaciones mantenidas con estos agentes clave configuran un tipo de diagnóstico actualizado sobre los límites y el potencial del territorio específicamente en un sector que es una de las principales fuentes de ingresos de su economía.

Metodológicamente, hemos utilizado entrevistas semiestructuradas, que, bajo una guía parcialmente estandarizada, permitían un desarrollo suficientemente libre para que los entrevistados desplegaran ideas sobre unas sugerencias temáticas (Català, 2016). Estas entrevistas fueron presenciales y realizadas entre el 28 de septiembre y el 23 de noviembre de 2015, normalmente en los puestos de trabajo de los individuos. En total se ha entrevistado a 33 personas.

En el conjunto de las organizaciones consultadas, están presentes tres ámbitos: empresarial, institucional y académico. Así, en el primer ámbito están representadas diferentes federaciones hoteleras de la zona (HOSBEC-Benidorm, Costa Blanca y Comunidad Valenciana; FEHM-Mallorca, FEHIF-Ibiza y Formentera, ASHOMEMenorca), de campings (federaciones catalana y de la Costa Brava), de apartamentos (Asociación de Alquiler de Apartamentos de Gandía), el Círculo Mallorquín de Negocios, hoteleros, regentes de campings y consultores privados en materia hotelera, de playas, de transportes y de turismo en general. En el ámbito institucional contamos con la presencia de los gobiernos de los tres territorios (Islas Baleares, Cataluña y Comunidad Valenciana), representados por la máxima autoridad político-administrativa en materia turística; también con los responsables de turismo de los consejos insulares de Ibiza y Menorca, el Patronato de Turismo de la Costa Brava y el Ayuntamiento de Peñíscola. Finalmente, el ámbito académico lo forman algunas de las universidades de estos territorios; en concreto, la Universitat de les Illes Balears (y su Escuela de Turismo de Ibiza), la Universitat d'Alacant, la Universitat de València, la Universitat Politècnica de València (y su Campus de Gandía), la Universitat Rovira i Virgili y la Escuela Superior de Administración y Dirección de Empresas (ESADE) de Barcelona. Estamos seguros que, a pesar de su limitación, se trata de una selección que puede representar adecuadamente el territorio para facilitarnos un diagnóstico de conjunto sobre el turismo sirviéndonos del análisis de discurso. 
Considerando las dos dimensiones del muestreo estructural, territorio y ámbito organizacional, que cuentan en ambos casos con tres categorías (las tres comunidades autónomas en el primer caso y los ámbitos empresarial, institucional y académico en el otro), las entrevistas quedan organizadas en 9 segmentos (empresarios catalanes, instituciones catalanas, académicos catalanes, empresarios baleares, etc.). Se ha garantizado un mínimo de 3 entrevistas por segmento y un mínimo de 9 por categoría. Esto ha permitido recoger voces suficientes en cada categoría y con la suficiente variedad para detectar discursos parciales de los diferentes colectivos. Pese al relativo equilibrio entre categorías (tanto de la dimensión territorial como la organizacional), las Islas Baleares están sobrerepresentadas para recoger adecuadamente las particularidades insulares de las tres islas principales, cada una de ellas con unas características específicas en cuanto a oferta y demanda turística, pero también en lo que atañe a relación con el medio (ver Riera y Aguiló, 2009).

A partir de estas entrevistas, se ha acometido un análisis de discurso en torno a los siguientes ejes: perfil de la demanda; la actividad complementaria al sol y playa; el problema de la estacionalidad; la competencia; las infraestructuras de transporte; la relación construcción-turismo; el problema de los apartamentos ilegales y su comercialización a través de portales de Internet; la transformación de los campings; y el empleo en el sector turístico. En el campo de los estudios turísticos, este tipo de análisis, prescrito como óptimo por Gutiérrez en su aproximación metodológica (2007: 186-188), ha sido aplicado en desarrollos de diferente índole, pero preferentemente para acometer el discurso de los demandantes o de los agentes de la oferta sobre aspectos tales como la elaboración de un diagnóstico, la fijación de retos y el dibujo de perspectivas sobre los destinos turísticos (Santana et al., 2014), que está en la línea de lo que aquí proponemos; el establecimiento de posiciones ideológicas en el sistema turístico residencial mediterráneo (Mantecón, 2011); o las diferentes propuestas que compilaron Jaworski y Pritchard (2005) desde un enfoque antropológico (discursos sobre turismo y cultura, la naturaleza en la experiencia turística, la implicación de los espacios turísticos sobre las identidades, etc.).

\section{DISCURSOS DE PRESENTE Y FUTURO SOBRE EL TURISMO DE COSTA}

En las líneas siguientes desarrollaremos, a través de diferentes puntos, la síntesis de lo que han aportado estos 33 agentes clave del territorio. Lo que resulta es un diagnóstico de situación y propuesta de líneas de actuación que pueden ser entendidas como políticas de desarrollo para el sector.

El inicio del turismo masivo en los sesenta y setenta, más el periodo de expansión 1998-2008, dominado por el negocio inmobiliario, han dado como resultado lo que hoy es el turismo litoral, con sus debilidades y sus fortalezas. Tanto la literatura académica, como los agentes clave del territorio y diferentes datos (Català, 2016), remiten a un periodo de gran intensidad constructora (1998-2008), que incluso algunos se han atrevido a calificar de depredación, porque ha cuestionado la capacidad de carga y el propio negocio turístico, muchas veces confundido con el inmobiliario. El compromiso del sector con un territorio que quiere ver preservado para mantener su atractivo natural y sociocultural, ha chocado con la rentabilidad a corto plazo de un capital inmobiliario que es, en esencia, transitorio respecto al espacio donde actúa, porque cuando acaba de consumir el territorio busca otro. La gran cantidad de vivienda construida, la amenaza sobre la sostenibilidad y la radicalidad de la caída una vez se 
produjo la crisis aconsejan no retomar estas sendas, y esto es una convicción que parecen tener desde todos los ámbitos (Administración, academia y empresa).

La sostenibilidad del territorio no está amenazada únicamente por el incremento de los flujos turísticos, sino por un aumento de la población en este periodo que ha contribuido a la densificación de muchas áreas litorales, como por ejemplo la Bahía de Palma, las Marinas o la Vega Baja en la provincia de Alicante, el área de SalouCambrils, etc. Las zonas turísticas acaban convertidas en áreas urbanas, y se intensifica el consumo de recursos. Para este aumento poblacional, se combina la inmigración laboral con el turismo residencial (en muchos casos, y proporcionalmente más en tiempos de crisis, jubilados europeos). A pesar de que el efecto metropolitano todavía tiene más capacidad de atracción residencial (especialmente en el caso de Barcelona y en primera residencia), el turismo ha hecho su contribución evidente $\mathrm{y}$, de hecho, los incrementos más notables de extranjeros se han producido precisamente en los principales destinos turísticos, señaladamente los que hemos mencionado, pero también en el Alt Empordà y las islas Pitiusas.

El paro ha afectado menos a los núcleos turísticos porque el sector ha aguantado la crisis mucho mejor que otras actividades, e incluso está creando ocupación directa e indirecta los últimos años. Esto también ha hecho que la dependencia del turismo sea cada vez mayor, especialmente en las Islas Baleares. Está claro que se tiene que insistir en la formación y la profesionalización del sector, pero la estacionalidad es un obstáculo que no se puede obviar, porque desincentiva las trayectorias formativas. La profundización en actividades complementarias puede crear nuevos puestos de trabajo y aumentar la calidad de los empleos directos de la hostelería, puesto que contribuyen a alargar la temporada. Por el contrario, se apunta que hay que perseguir la oferta ilegal, precisamente porque no crea trabajo, e intentar convertir los apartamentos en hoteles, porque esto contribuirá a la calidad global del destino y al empleo. Asimismo, la Administración tiene que perseguir las malas prácticas laborales, más aun teniendo en cuenta que el sector está poco sindicado.

Por otro lado, hay que aprovechar la experiencia en el sector y la capacidad de un parque alojativo muy extenso, tanto en apartamentos y hoteles (con el liderazgo de la isla de Mallorca, cuyos empresarios se han expandido internacionalmente, Benidorm y la hotelería urbana de Barcelona), como en campings (con grandes concentraciones en el Alt Empordà, prolongadas a la Costa Roja, ya en territorio francés). Es una oferta conjunta la de este territorio que no tiene comparación en todo el Mediterráneo. Se ofrece un volumen difícil de igualar con una relación calidad/precio que tiene poca competencia. De todas maneras, se asume que hay que huir de la autocomplacencia y estar atentos a un mercado que ahora sopla a favor por la ausencia de seguridad en el Mediterráneo sur y Oriente Próximo, pero que presentaba una amenaza concretada en destinos como Túnez o Egipto, que, si superan este momento difícil, pueden volver a atraer parte de la cuota que ahora ostenta el Mediterráneo español (precisamente, los atentados de Barcelona pueden haber contribuido a reequilibrar la distancia). Del mismo modo, en la parte oriental de Europa (Balcanes, Grecia, Chipre o Turquía) hay un retraso infraestructural que poco a poco pueden ir superando, singularmente Croacia (ya con un volumen de oferta importantísimo) y Turquía (que está construyendo hoteles de alto nivel). Nuevamente, el desarrollo de la oferta complementaria es la vía para dar solución a los retos que se plantea el sector, porque si los destinos consiguen este valor añadido, sufrirán menos fugas de clientes hacia potenciales destinos estabilizados y desarrollados. 
Asimismo, campings y hoteles tienen que seguir apostando por la calidad, por la adecuación y modernización de su infraestructura, así como por los incrementos de categoría, que están concentrando una amplia mayoría de hoteles de tres y cuatro estrellas en prácticamente todas las marcas turísticas. En paralelo, la singularización de la oferta de alojamientos también es una herramienta para la consolidación dentro de un mercado tan competitivo, y por eso la tematización, la especialización, la incorporación de nuevos elementos a la oferta (como por ejemplo las casas en árboles en los campings o cualquier atractivo que amplíe la oferta a la vez que la diversifique) son caminos en los que es interesante profundizar. Los hoteles y residencias ligados a la salud son un ejemplo claro, pero también la tematización musical de los hoteles en Ibiza, cuestión que se ha convertido en el último desarrollo de un posicionamiento de alcance internacional, como nos comenta una de las entrevistadas:

\begin{abstract}
En nuestro caso, en Ibiza, somos referencia mundial de vanguardia en ocio musical, y como paso más allá dentro de esto, la implementación de los hoteles musicales. En el plan estratégico 2005-2010 de Ibiza se decía que el producto por excelencia es el sol y playa, evidentemente, pero ya iba viéndose en la matriz que la posición del sol y disco estaba casi al mismo nivel. ¿Qué implicó esto? Pues, a pesar de que ya se sabía, se despertó definitivamente el reforzamiento de esta línea, con los hoteleros de aquí poniendo el foco en el desarrollo de establecimientos de alojamiento que recogieran el concepto. (...) El apelativo de musical [para los hoteles] implica que tú estás viviendo la experiencia musical siempre, con repertorios musicales continuados, fiestas, los mejores DJ del mundo, conciertos, beach clubs propios, etc. (...) Se trata de una diferenciación de producto absolutamente exitosa y de gran rentabilidad, con un grado de sofisticación elevado y ofreciendo exclusividad al cliente. (...) Y los beach clubs exclusivos forman parte de esto también. (...) Ibiza es marketing puro, es un parque temático de la exclusividad. (...) Y en el tema musical parece que para que una cosa sea tendencia y tenga éxito mundial, debe tener éxito primero en Ibiza, que funcione aquí (Yolanda Ortiz, Escuela de Turismo de Ibiza).
\end{abstract}

En este sentido, los resorts pueden participar de este tipo de estrategias, en tanto que muchas veces el complejo turístico que forman se articula alrededor de un tema. Las entrevistas han servido para contraponer diferentes visiones sobre el encaje de esta modalidad en la zona: ¿pueden tener el mismo éxito aquí que en el Caribe o en la Polinesia? Es evidente que la diversidad de producto del Mediterráneo este español (ya disfrutamos de una "tematización mediterránea") puede retardar su avance, aunque la extensión de esta modalidad en todo el mundo es una tendencia difícil de combatir, sobre todo si el capital hotelero apuesta por ello. Pero precisamente es una modalidad que tiene poco impacto sobre la economía local, en tanto que se suele pagar un todo incluido en los lugares de origen a mayoristas y grupos que no suelen ser de capital autóctono, y después el cliente apenas sale del recinto (y, por lo tanto, hace pocas aportaciones extra a la economía). Otra cuestión, quizá, son los resorts o parques de ocio asociados a la modalidad de camping, que suelen tener su fundamento en el contacto con la naturaleza y, de este modo, las salidas son frecuentes (aparte de que los campings suelen ser de capital local). Los entrevistados apuntan que, en lo posible, habría que estar pendientes del tipo de resorts que se instalan y buscar convenios para actividades externas.

En la modalidad de apartamentos, incide un problema antiguo amplificado con herramientas nuevas; es decir, desde siempre han existido los apartamentos ilegales, gestionados si acaso por porteros de las urbanizaciones o alguna otra figura que se presta a la irregularidad; pero Internet y plataformas especializadas como por ejemplo Airbnb o Home Away, por mencionar las dos que tienen una mayor cuota en España (Ernst \& Young, 2015), están intensificando la competencia e incluso provocando migraciones de apartamentos regularizados a ilegales, porque los propietarios ven más 
expectativa de negocio. Es justamente en tiempos de crisis cuando estas plataformas han proliferado más, porque, sin las obligaciones impositivas o de adaptación a las normativas, se pueden ofrecer precios más bajos y es un alternativa barata de viaje (un apartamento Airbnb, combinado con Blablacar o las compañías aéreas de bajo coste, permiten la autoconfección de paquetes de viaje muy económicos). La modalidad regularizada ya había aumentado en el inicio del período de crisis, en una gráfica de evolución prácticamente inversa a la de los hoteles, pero en este caso, el de los apartamentos ilegales, el crecimiento ha sido exponencial, y afecta a todos los grandes destinos turísticos litorales, con proporciones próximas al $50 \%$ en núcleos como Peñíscola o Lloret de Mar y más todavía en destinos urbanos como Barcelona, Palma o Valencia (Ernst \& Young, 2015: 9-13). Independientemente de que este tipo de economía sumergida se adapte al bolsillo de una clase media empobrecida, a diferentes cambios de hábitos ligados a la tecnología y la economía colaborativa, y dé salida al excedente inmobiliario, los inconvenientes superan con creces a las ventajas, al menos desde una perspectiva global del sector. A la escasa contribución a la creación de empleo y de valor de los apartamentos no regularizados, se añaden problemas como por ejemplo la confusión entre las zonas y los usos de primera y segunda residencia, el beneficio para la empresa transnacional y no para la local, la ausencia de protección de un cliente que no puede reclamar (fuera de escribir una mala opinión en el portal de Internet y contribuir a que le vaya peor al propietario), la dificultad para los trabajadores de encontrar alquiler a precios asequibles, porque los arrendatarios acaban uniéndose a estas plataformas y al mercado turístico sumergido, en los que encuentran más rentabilidad; la merma global de la calidad del destino, etc. Lo que se hace necesario en relación a este fenómeno, y es lo que pide tanto el sector como la Administración, es la revisión de la normativa para situar claramente en la ilegalidad estas actividades y obligar a los propietarios a pasar a la regularización (de este modo, harían las obras para adaptarse a las normativas, pagarían impuestos, se incorporarían a una recepción conjunta, etc.), pero también destinar recursos a una inspección que vele porque todos los alquileres sean legales; intervenir en la ordenación espacial de la oferta, para no generar problemas de convivencia entre residentes y vacacionistas; y calificar las plataformas como canal de oferta turística para que estén regidas por la normativa vigente que, a su vez, los obligaría al control de su oferta (para un mayor detalle sobre propuestas en esta materia, véase Ernst \& Young, 2015: 43-59).

En otro orden de cosas y en contraste con lo que sucede en otros sectores, hemos podido comprobar que los agentes clave del turismo son bastante mesurados en las demandas de infraestructuras del transporte, seguramente en la línea de esta apuesta por la sostenibilidad. De hecho, en las Islas Baleares ninguna infraestructura de transporte es considerada prioridad máxima, pero, en cambio, sí que lo son las de abastecimiento y depuración de agua, que son cuestión central para las administraciones baleares. En el litoral peninsular sí que es una prioridad de primer orden la finalización del corredor ferroviario mediterráneo, en consonancia con lo que piensan en otros sectores. También se ve interesante, aunque con una posición jerárquica inferior, ampliar y mejorar la red ferroviaria secundaria, y el foco se ha puesto especialmente en el tramo de costa ElcheGandia, en buena medida para conectar con el aeropuerto de Alicante-Elche. En cuanto al modo aéreo, se renuncia de plano a cualquier ampliación de los aeropuertos, pero se considera necesario un incremento de las conexiones, especialmente fuera de temporada y a las islas pequeñas, porque la ausencia de líneas actúa como obstáculo para la desestacionalización (o, más bien, para el alargamiento de la temporada); y mejorar los accesos a los aeropuertos siguiendo una lógica intermodal. El modo viario es el menos prioritario, a pesar de que los valencianos demandan algunas actuaciones en altas 
prestaciones (gratuidad de la AP-7, finalización de la autovía CV-10 en el prelitoral de Castellón, una entrada de autovía para Calp). Y tampoco se apuntan demasiadas intervenciones en el modo marítimo, a pesar de que en las Islas Baleares, el territorio que, lógicamente, más lo necesita, son proclives a hacer mejoras (en Ciutadella, en Ibiza, en Alcúdia...). En la ausencia de entusiasmo con este modo, influye el hecho que no se vea demasiado rendimiento a la llegada de cruceros (salvo el caso de Barcelona, que sí ejerce como base de muchos de ellos, algunos de los cuales entre los más grandes del mundo).

Precisamente la rentabilidad de los destinos turísticos, su capacidad para sacar provecho del desarrollo de la actividad turística, es una de las cuestiones sobre las cuales el sector está pendiente. En este sentido, parece haber una diferencia entre las Islas y Cataluña, por un lado, y la Comunidad Valenciana, por el otro. De entrada, la renta y el PIB per cápita de los valencianos están muy por debajo de las cifras de catalanes y baleares, y esto debe tener un traslado en los precios y en las tasas de ganancia. Pero el caso es que los ingresos medios por habitación disponible son más elevados en las Islas y en Cataluña. Además, el gasto que hacen tanto los extranjeros como los autóctonos es mayor en estos territorios. Cierto es que el núcleo turístico principal de la Comunidad Valenciana, Benidorm, que concentra más del $40 \%$ de las pernoctaciones en tierras valencianas, es el ejemplo más próximo a la desestacionalización, y que esto lo consigue tomando buena parte del segmento sénior y bajando mucho los precios (normalmente sacando un beneficio nulo o escaso), y esto repercute en las cifras finales, que no estarían tan lejos de las catalanas o baleares si no fuera por este hecho. Pero desde la Generalitat Valenciana también se reconoce un problema de rentabilidad, que se tiene que afrontar aumentando la calidad y apostando por el valor añadido en los destinos turísticos. En cualquier caso, y como apuntaba el profesor Antoni Riera (UIB), nada de esto aportará bienestar a la población si continúa dominando el patrón de acumulación, en lugar del de aprovechamiento.

Por otro lado, si ya hemos hablado de la globalización en términos de competencia, porque hoy más que nunca la competencia es internacional (el Mediterráneo español puede competir con las islas del Egeo o con la costa adriática, pero el Mediterráneo también compite globalmente con el Caribe o con los mares de Australasia), la dimensión global está presente en todos los grandes retos que afectan a la población, pero también de manera muy clara al turismo, como por ejemplo la inseguridad (protagonizada en estos momentos sobre todo por el terrorismo islamista), el cambio climático, la uniformización cultural, las consecuencias del desarrollo de las TIC (especialmente en cuanto al cambio de hábitos y el uso de Internet y APP en todas las fases de la experiencia turística), la disponibilidad de cada vez más vuelos y conexiones... Esto último implica que los clientes pueden irse a otros destinos, pero también que pueden desplazarse aquí turistas de lugares más lejanos y que hasta hace poco apenas venían. Por lo tanto, el turismo de estas costas tiene que procurar conservar los actuales visitantes, pero explorar la posibilidad de atraer nuevos, especialmente si aprecian la oferta complementaria y están dispuestos a pagar más de lo que pagan por promedio los actuales. Además, el momento actual todavía es de dominio de la touroperación clásica, pero va a menos y se incorporan con fuerza plataformas de Internet e intermediarios más especializados que añaden complejidad al mercado. También hemos hecho referencia a la homogeneización inherente al aumento de los intercambios a escala planetaria y, en esto, lo que les ocurre a los destinos turísticos es causa y consecuencia a la vez, porque los hoteles (y más los de capital transnacional) y otros espacios públicos ofrecen una imagen uniforme que nos socializa a todos en este 
tipo de escenarios (más todavía si se trata de un resort, que limita el contacto con las particularidades del territorio); pero también los turistas de diferentes lugares que llegan a los destinos contribuyen a esta homogeneidad, porque los espacios acaban adaptándose a ellos y ofreciendo una imagen reconocible, que les ofrezca cierta seguridad, a pesar de que sea compatible con los particularismos locales. La interacción de los turistas con los autóctonos contribuye al enriquecimiento mutuo, pero también a aportar elementos ajenos a la cultura autóctona.

Es cierto que la era del turismo masivo y de los turoperadores nos dejó un consumidor afecto a los lugares comunes y seguros, al viaje organizado. En cierto modo era así, porque en los primeros momentos (los sesenta, los setenta, e incluso los ochenta) este tipo de viaje era una novedad, un reflejo de la modernidad. Pero, una vez superada esta fase y con el dominio de los espacios de uniformidad, poco a poco el turista está demandando justamente lo contrario; está demandando autenticidad, ausencia de aculturación, conservación del entorno. $\mathrm{Y}$ ésta es la clave de la transformación que está sufriendo ya desde hace más de una década el turismo litoral en el Mediterráneo noroccidental, la apuesta por el turismo sostenible en sus vertientes medioambiental, sociocultural y económica (la sostenibilidad en el plano sociocultural quiere decir que debe mantenerse la forma de vida local y que no se haga del destino un espectáculo, sino respetar la función que cumplen los recursos y los activos turísticos, más todavía si tienen un sentido dentro de la economía local). Seguramente, éste es el asunto en que la literatura académica sobre el turismo está haciendo más hincapié en los últimos años y también es la cuestión que más ha aflorado en el discurso de los agentes clave en este trabajo, más aún como contraste con la fase anterior en la que se puso el foco en la construcción y no en la experiencia turística. Administración y empresas deben colaborar para activar el patrimonio natural y cultural que permita la actividad complementaria que demanda un turista que, crecientemente, quiere algo más que pasarse todo el tiempo en la playa ${ }^{11}$ o tener una rutina gastronómica estándar, sino que también quiere hacer una ruta en bicicleta de montaña; quiere probar los vinos locales; disfrutar de la arquitectura de un pueblo pescador; comer en alguno de los restaurantes que destaca Tripadvisor, Vendimia, la Guía Michelin o cualquier otro portal prescriptor; recorrer la amplia red de pequeño comercio de lugares como Benidorm o Calvià, o ir de shopping a Palma, Barcelona, Valencia o Ibiza; hacer submarinismo en una cala de Menorca; visitar los restos de Tarraco; ir a pasar un día en Port Aventura o a uno de los parques de atracciones de Benidorm; y un amplio etcétera. Las playas continuarán siendo un ingrediente fundamental, y se entiende que su preservación en el mejor estado posible ha de ser una prioridad: el más cercano al original en los lugares no urbanos, y de una forma digna si se trata de playas urbanas, que tienen que permanecer limpias, pero dotadas de servicios. Pero junto a las playas, hay que cuidar el resto del entorno natural para que la experiencia de hacer actividades al aire libre sea lo más satisfactoria posible. Y una de las virtudes de esta oferta complementaria es que, donde está teniendo un desarrollo mayor, se produce el alargamiento de la temporada y la diversificación del perfil del turista. En este sentido, y mezclado entre un turismo familiar que todavía es dominante, va incorporándose un turismo de pareja o de grupos de amigos que no tienen como finalidad principal el ocio nocturno (por ejemplo, que vayan una semana a Ibiza a hacer submarinismo o vela y sólo el último día lo destinen a ir de fiesta a un night-club de la playa de En Bossa), que se ha comprobado que hacen un gasto medio con impacto sobre la economía local muy por encima de la media, y justamente es así por la

${ }^{11} \mathrm{Y}$ ya hemos visto que algunos de los nuevos mercados que se están intentando atraer, como por ejemplo, el chino, ni siquiera van a la playa. 
diversidad de producto que consumen y por el nivel de exigencia de sus vacaciones. A pesar de que también hay turistas autóctonos bajo esta modalidad, es más habitual que sean extranjeros, y es en lugares como Menorca donde se ha detectado más claramente su proliferación.

\section{CONCLUSIONES}

En definitiva, el repaso a las cuestiones abordadas por los entrevistados nos ofrece el planteamiento de un modelo para el turismo litoral que deja atrás definitivamente la época de la expansión inmobiliaria; que apuesta por el turismo sostenible como principio básico (medioambiental, sociocultural y económicamente); que conserva las playas en el estado más parecido al original en las no antropizadas y, en el caso de las playas urbanas, mantiene la limpieza y la dotación de servicios; y que se ponen las herramientas necesarias para pasar de un patrón de acumulación a un patrón de aprovechamiento, que posibilite que, en último extremo, se distribuya la riqueza que está generándose gracias al turismo (Riera y Aguiló, 2009: 177). Pero también hay que estar atentos a los cambios del mercado para avanzarse a las consecuencias que éstos tengan, especialmente en los países de origen y en las potencialidades de las tecnologías de la información y la comunicación para absorber el máximo de cuota de la cadena de valor. También queda claro que se tiene que poner en valor el patrimonio natural y cultural para fortalecer la oferta complementaria, que se debe entender como un factor de positivación múltiple: creación de trabajo, incremento del atractivo y competitividad del destino, atracción de nuevos segmentos de mercado, defensa ante los competidores, alargamiento de la temporada, etc. En esta línea, la singularización de la oferta alojativa se ha revelado como una de las vías para la consolidación y el crecimiento de los destinos. Además, hay que continuar en la línea de la profesionalización del sector siguiendo el modelo del subsector gastronómico, que en algunas zonas va logrando niveles de excelencia; apostar de forma decidida por la calidad y la innovación, dejando aparte la expansión de la oferta; y dar una salida satisfactoria a la proliferación de oferta ilegal en los términos que han quedado expuestos. La coyuntura favorable tiene que servir, lejos de caer en la autocomplacencia, para profundizar en el camino del incremento de la calidad, en la vía de la profesionalización del sector y en la atenuación de los efectos de este periodo de expansión inmobiliaria (empezando, lógicamente, por aprender de los errores).

\section{BIBLIOGRAFÍA}

ALEDO TUR, A. De la tierra al suelo: la transformación del paisaje y el nuevo turismo residencial. En: Arbor-Ciencia, Pensamiento y Cultura. 2008, enero-febrero, $\begin{array}{lllll}\text { CLXXXIV, } & \mathrm{n}^{\circ} & \text { 729, } & \text { 99-113. }\end{array}$ http://arbor.revistas.csic.es/index.php/arbor/article/view/164/164.

ANTON CLAVÉ, S. El consum d'experiències i emocions. En: Paradigmes. 2009, 3, pp. 93-100. http://www.raco.cat/index.php/Paradigmes/article/viewFile/225295/306603. ANTON CLAVÉ, S. Identitat i turisme. Entre la imatge i la percepció. En: Paradigmes: Economia Productiva $i$ Coneixement. 2010, $\mathrm{n}^{\mathrm{o}}$ 5, pp. 157-165. http://www.raco.cat/index.php/Paradigmes/article/view/218429/297577.

BARCELÓ HORRACH, C. (dir.). Estudio sobre productos emergentes en las Illes Balears, Palma de Mallorca: Cambra de Comerç de Mallorca, 2010. 
BURRIEL DE ORUETA, E. L. Los límites del planeamiento urbanístico municipal. El ejemplo valenciano. En: Documents d'Anàlisi Geogràfica. 2009, no 54, pp. 33-54. https://ddd.uab.cat/pub/dag/02121573n54/02121573n54p33.pdf_error_estat_espanyol.

BURRIEL DE ORUETA, E. L. El estallido de la burbuja inmobiliaria y sus efectos en el territorio. En ALBERTOS PUEBLA, J. M. y SÁNCHEZ HERNÁNDEZ, J. L. (coords.). Geografía de la crisis económica en España. València: Universitat de València, 2014, pp. 101-140.

BUTLER, R. W. The concept of a tourism area cycle of evolution: implications for management and resources. En: The Canadian Geographer. 1980, $\mathrm{n}^{\mathrm{o}}$ 1, pp. 5-12. https://www.researchgate.net/publication/228003384_The_Concept_of_A_Tourist_Are a_Cycle_of_Evolution_Implications_for_Management_of_Resources.

CAMBRILS CAMARENA, J. C. y NÀCHER ESCRICHE, J. M. Reposicionamiento en destinos maduros. El caso de Gandía. En: Estudios Turísticos. 2005, n ${ }^{\circ} 166$, pp. 115131. http://estadisticas.tourspain.es/img-iet/revistas/ret-166-2005-pag115-13194698.pdf.

CARVALHO, K. D. y MOQUETE GUZMÁN, S. J. El turismo en la dinámica territorial. ¿Lógica global, desarrollo local?. En: Estudios y Perspectivas en Turismo. 2011, vol. 20, no 2, pp. 441-461. http://www.redalyc.org/articulo.oa?id=180717607010.

CATALÀ OLTRA, L. El turisme a l'EURAM. La xarxa de costa. València: Institut Ignasi Villalonga d'Economia i Empresa, 2016. http://euroregioeuram.eu/new/media/Elturisme-a-LEURAM-la-xarxa-de-costa.pdf.

CÓRDOBA LEIVA, J. L. La revolución silenciosa del turismo. En: International Journal of World of Tourism. 2014, vol. 1, n⿳⺈ 1, pp. 9-16.

CREUS, M. y CELADA PRIOR, G. ¿Que vienen las suecas! Una aproximació estètica, simbòlica, social i política al turisme massiu de sol i platja. En: Quadern de les Idees, les Arts i les Lletres. 2011, n 182, pp. 5-9.

https://dialnet.unirioja.es/servlet/articulo?codigo $=3869878$.

DEMAJOROVIC, J.; ALEDO TUR, B. LANDI, A. y MANTOVANI KONDO, A. L. Complejos turísticos residenciales. Análisis del crecimiento del turismo residencial en el Mediterráneo español y en el Litoral Nordestino (Brasil) y su impacto socio-ambiental. En: Estudios y Perspectivas en Turismo. 2011, vol. 20, pp. 772-796. http://www.redalyc.org/articulo.oa?id=180722696002.

ERNST \& YOUNG. Alojamiento turístico en viviendas de alquiler: impactos y retos asociados. Madrid: Exceltur, 2015.

http://www.exceltur.org/wp-content/uploads/2015/06/Alojamiento-tur\%C3\%ADsticoen-viviendas-de-alquiler-Impactos-y-retos-asociados.-Informe-completo.-Exceltur.pdf. FERNÁNDEZ MUÑOZ, S. y BARRADO TIMÓN, D. A. El desarrollo turísticoinmobiliario de la España mediterránea e insular frente a sus referentes internacionales (Florida y la Costa Azul): un análisis comparado. En: Cuadernos de Turismo. 2011, no 27, pp. 373-402. https://digitum.um.es/xmlui/bitstream/10201/28019/1/140011-5310311-SM.pdf.

FLORES ALBERTO, D.; SALAZAR NIEBLA, L. y SANTANA TURÉGANO, M. A. ¿Desaparecerán los Tour Operadores? El papel de los intermediarios en la distribución turística: análisis del caso de Tenerife. En: Pasos: Revista de Turismo y Patrimonio Cultural. 2011, vol. 9, nº 2, pp. 341-159.

http://www.pasosonline.org/Publicados/9211/PS0211_10.pdf.

GUTIÉRREZ BRITO, J. (coord.). La investigación social del turismo. Madrid: International Thompson, 2007.

GUTIÉRREZ TAÑO, D. Las actitudes de los residentes ante el turismo. San Cristóbal de la Laguna (Tenerife): Universidad de La Laguna, 2010. 
HORRACH ESTARELLAS, B. Nuevas pautas de regeneración de los destinos turísticos maduros. Tras más de cien años de construcción de Platja de Palma. En: Arquitectura, Ciudad y Entorno. 2014, vol. 9, no 25, pp. 349-372.

http://upcommons.upc.edu/bitstream/handle/2099/14913/3631-523-2-

RV.pdf?sequence $=7$ \&is Allowed $=\mathrm{y}$.

HOSTELTUR. El sol y playa, ¿un modelo estancado o estabilizado?. En: Hosteltur. 2004, otoño, pp. 6-40.

http://www.uhu.es/uhutur/documentos/npturisticos2/Turismo\%20de\%20sol\%20y\%20pl aya.pdf

IGLESIAS GARCÍA, M. y FERNÁNDEZ POYATOS, M. D. Las redes sociales en las marcas turísticas de la Comunidad Valenciana. En: Estudios sobre el Mensaje Periodístico. 2012, vol. 18, noviembre, pp. 533-541.

https://rua.ua.es/dspace/bitstream/10045/26997/1/ArticleEMP2012xarxesComplet.pdf. INNOVA MANAGEMENT. Plan de marketing turístico de Benidorm, 2011-2015, Benidorm (Alicante): Fundación Turismo de Benidorm, 2010. https://es.slideshare.net/juansobejano/plan-marketing-benidorm.

IVARS i BAIDAL, J. A.; RODRÍGUEZ SÁNCHEZ, Isabel y VERA REBOLLO, J. F. The evolution of mass tourism destinations: new approaches beyond deterministic models in Benidorm. En: Tourism Management. 2013, vol. 34, febrero, pp. 184-195. https://rua.ua.es/dspace/bitstream/10045/34318/3/2013_Ivars_etal_TourismManagemen t corrected proof.pdf.

JAWORSKI, A. y PRITCHARD, A. (eds.). Discourse, communication and tourism, Clevedon (Reino Unido): Channel View Publications, 2005.

MANTECÓN, A. El proceso del turismo residencial: análisis sociopolítico de los discursos públicos desde una perspectiva cualitativa. En: Empiria: Revista de Metodología de Ciencias Sociales. 2011, 21, pgs. 17-38.

http://revistas.uned.es/index.php/empiria/article/view/858/781.

MARCO LAJARA, B.; GARCÍA LILLO, F., SABATER SEMPERE, V. I y UBEDA GARCÍA, M. Impacto del territorio en la rentabilidad de los hoteles vacacionales españoles. Un análisis comparativo de las principales comunidades autónomas y puntos turísticos de la costa mediterránea y archipiélagos canario y balear. En: Análisis Turístico. 2011, $\mathrm{n}^{\mathrm{o}}$ 12, segundo semestre, pp. 70-78.

http://www.aecit.org/jornal/index.php/AECIT/article/view/108/100.

NAVALÓN GARCÍA, R. y RICO CÁNOVAS, E. Estrategias de futuro para el destino tradicional de la Costa Blanca: el Plan de valorización turística del patrimonio cultural de la provincia de Alicante. . En: XII Coloquio de Geografía del Turismo, Ocio y Recreación: espacios y destinos turísticos en tiempos de globalización y crisis. Universidad Carlos III, Colmenarejo (Madrid), 2011. https://earchivo.uc3m.es/bitstream/handle/10016/16483/estrategias_navalon_rico_TERAP_201 1 .pdf.

NOVO ESPINOSA DE LOS MONTEROS, G. et al. Viajes, actuantes, escenarios e interacciones: un análisis de la publicidad turística de los destinos, a partir de sus semánticas visuales. En: Investigaciones Turísticas. 2013, nº 6, julio-diciembre, pp. 2746.

https://rua.ua.es/dspace/bitstream/10045/34777/1/Investigaciones_Turisticas_6_02.pdf. OBIOL MENERO, E. y PITARCH GARRIDO, M. D. El litoral turístico valenciano. Intereses y controversias en un territorio tensionado por el residencialismo. En: Boletín de la Asociación de Geógrafos Españoles. 2011, $\mathrm{n}^{\mathrm{o}}$ 56, pp. 177-200. 
http://roderic.uv.es/bitstream/handle/10550/40753/068239.pdf?sequence=1\&isAllowed $=\mathrm{y}$.

OMT-Organización Mundial del Turismo. Panorama OMT del turismo internacional, Madrid: OMT, 2017. https://www.e-unwto.org/doi/pdf/10.18111/9789284419043.

PÉREZ SOSA, I. y NAVAS FERRER, T. Evolución histórica de dos destinos turísticos internacionales. Maspalomas-Costa Canaria y Costa Brava. En: Arquitectura, Ciudad y Entorno. 2014, vol. 9, nº 25, pp. 229-278.

http://upcommons.upc.edu/bitstream/handle/2099/14909/3627-515-2-

RV.pdf?sequence $=7 \&$ is Allowed $=\mathrm{y}$.

RAMOS CARAVACA, C. Costa Brava, los retos urbanísticos del turismo de masas: la huella de la ciudad jardín y algunos principios racionalistas en el tejido turístico de masas. En: Identidades: Territorio, Cultura, Patrimonio. 2013, n 4, pp. 155-189. http://upcommons.upc.edu/bitstream/handle/2117/82126/6.pdf?sequence=1\&isAllowed $=\mathrm{y}$.

RIERA FONT, A. y AGUILÓ PÉREZ, E. (dirs.). Llibre blanc del turisme de les Illes Balears. Cap a una nova cultura turística. Palma de Mallorca: Govern de les Illes Balears-Universitat de les Illes Balears-"Sa Nostra" Caixa de Balears-Cambra de Comerç de Mallorca, 2009.

ROCA BLANCH, E. Urban intervention on the coastline of Catalunya. A new understanding of tourism. En: Arquitectura, Ciudad y Entorno. 2014, vol. 9, no 25, pp. 327-348. $\quad$ http://upcommons.upc.edu/bitstream/handle/2099/14912/3630-521-2RV.pdf?sequence $=7 \&$ is Allowed $=y$.

ROCA BOSCH, E. y VILLARES JUNYENT, M. Reforzar la resiliencia socioecológica de los destinos turísticos. En: Arquitectura, Ciudad y Entorno. 2014, vol. 9, no 25, pp. 493-524. $\quad$ http://upcommons.upc.edu/bitstream/handle/2099/14919/3637-535-2RV.pdf?sequence $=7 \&$ is Allowed $=\mathrm{y}$.

ROVIRA SOTO, M. T. y ANTON CLAVÉ, S. De destino a ciudad. La reformulación urbana de los destinos turísticos costeros maduros. El caso de la Costa Daurada central. En: Arquitectura, Ciudad y Entorno. 2014, vol. 9, $\mathrm{n}^{\mathrm{o}}$ 25, pp. 373-392. http://upcommons.upc.edu/bitstream/handle/2099/14914/3632-525-2-

RV.pdf?sequence $=7 \&$ is Allowed $=\mathrm{y}$.

RUIZ MAFÉ, C. y TRONCH GARCÍA DE LOS RÍOS, J. Influencia de las motivaciones hedónicas en el valor percibido de las web 2.0 de alojamientos turísticos. En: Gran Tour: Revista de Investigaciones Turísticas. 2013, $\mathrm{n}^{\mathrm{o}}$ 7, enero-junio, pp. 4-22. http://roderic.uv.es/bitstream/handle/10550/40499/093497.pdf?sequence=1\&isAllowed $=\mathrm{y}$.

SANTANA, A.; RODRÍGUEZ, A. J. y DÍAZ, P. El análisis cualitativo en el diagnóstico del ciclo de vida de áreas turísticas. En VVAA, Periferias, fronteras y diálogos. Tarragona: Universitat Rovira i Virgili, 2014, pgs. 2266-2295. https://www.researchgate.net/publication/266674272_El_analisis_cualitativo_en_el_dia gnostico_del_Ciclo_de_Vida_de_Areas_Turisticas_El_caso_del_Puerto_de_La_Cruz_ Tenerife_Islas_Canarias.

SANTOS, M. C.; FERREIRA, A. M. y COSTA, C. Influential factors in the competitiveness of mature tourism destinations. En: Tourism \& Management Studies. 2014, 10 (1), pp. 73-80. https://dialnet.unirioja.es/descarga/articulo/4788840.pdf.

TORRES SOLÉ, T.; SALA RÍOS, M. y FARRÉ PERDIGUER, M. O turismo sostible nas marcas turísticas de Cataluña. En: Revista Galega de Economía. 2013, vol. 22, nº 1, junio, pp. 51-72. http://www.redalyc.org/pdf/391/39127027003.pdf.

UNEP-Programa de las Naciones Unidas para el Medioambiente. Green Economy and Trade. Trends, Challenges and Opportunities, Nairobi (Kenia): UNEP, 2013. 
http://www.greengrowthknowledge.org/sites/default/files/downloads/resource/GE_and trade\%20-\%20trends_challenges_opportunities_UNEP.pdf. 